\title{
Análise da integração da modelagem gerativa com BIM: interoperabilidade, potenciais e fluxo do processo no par Revit ${ }^{\circledR}$ - Dynamo.
}

\author{
Integration analysis of generative modeling with BIM: interoperability, potentials and flow \\ process with the pair Revit@ - Dynamo.
}

\author{
Douglas Lopes de Souza \\ Universidade Federal de Viçosa, Brasil \\ douglas@ufv.br \\ André Teixeira da Costa \\ Universidade Federal de Viçosa, Brasil \\ andre.t.costa@ufv.br
}

\author{
Andressa do Carmo Pena Martinez \\ Universidade Federal de Viçosa, Brasil \\ andressamartinez@gmail.com \\ Denise Mônaco dos Santos \\ Universidade Federal de Viçosa, Brasil \\ denise.monaco@ufv.br
}

\begin{abstract}
The practice of generative modeling had a great expansion in recent years and great potential linking systems and software in order to increase the optimized production of their morphologies. In this panorama, BIM processes has a great impact in maintaining consistency between the data generated, the coordination process and the object constructability. This paper presents an analysis of different cases with algorithms applied in different optimization goals proposing an analysis feature of interaction between models and predicting the possibility of structuring a dynamic and interoperable process.
\end{abstract}

Keywords: BIM; Modelagem integrada; Modelagem Gerativa; Processo de projeto; Interoperabilidade.

\section{Introdução}

Nos últimos anos vimos a declaração do potencial da simulação performática e da morfogênese como resultado de um fenômeno recursivo que tem produzido o aumento do interesse por formas mais complexas e modelos conceituais mais avançados formal e performaticamente. Devido à natureza de seu processo recursivo que, de acordo com Bentscheff e Gengnagel (2010), o modelo opera pela informação do sistema com dados de outras disciplinas e sua consolidação em forma é a real condição de sua complexidade.

Como apresentado por Schumacher (2008), o uso de parametrização ou de modelagem baseada em scripts de programação, permite que a concepção do modelo manifeste a diferenciação e adaptação nos elementos constituintes do edifício, seus subsistemas ou em cada uma de suas alternativas. É importante que este processo seja interativo e não relegado ao formalismo porque seu potencial é determinado pela coesão entre o pensamento da concepção e da construção, permitindo uma atitude que retira o arquiteto de uma prática autônoma e retoma o conceito de produção multidisciplinar por meio da simulação e a efetivação de resultados nas diversas expressões do objeto. No paradigma atual, os softwares de design paramétrico são absorvidos por diversas razões tal como a popularização do pensamento algorítmico simplificado pela interface visual de programação. Mas a grande vantagem deste pensamento reside na linguagem aberta que tem permitido a emergência de plugins, como os de simulação física e evolutiva.

\section{Morfogênese e performance}

Apesar do avanço tecnológico que permitiu este tipo de abordagem, o processo de projeto que se dedica a esta modelagem facilmente recai sobre 0 expressionismo estrutural iniciado nos anos 70 que possui dentro de seus representantes o World Trade Center, de Minoru Yamasaki, 1971, Lloyd's Building de Richard Rogers, 1986, Centro Georges Pompidou, do mesmo arquiteto em conjunto com Renzo Piano e aos exemplos mais contemporâneos como as obras dos arquitetos Santiago Calatrava, Norman Foster, Zahad Hadid, dentre outros.

No modelo de projeto apresentado por Schumacher (2012), o processo depende da organização espacial e da performance técnica que também pode ser dada a partir de uma articulação material. A falácia que é gerada pelo uso da modelagem gerativa neste tipo de processo é a materialização de uma morfologia tecnicamente adequada que usa este caráter na sua justificativa, determinando uma morfologia com fim em si mesma ao invés de ser um meio para articular a função social do artefato no espaço em questão.

A este uso estratégico de um elemento tecnicamente induzido na morfologia com objetivo de articular e direcionar funções sociais, Schumacher (2012) o nomeia como uma operação tectônica. Como resultado deste processo podemos encontrar diversas soluções como a diferenciação adaptativa de estruturas, volumes e envoltórias de acordo com a performance do edifício de modo a permitir diferentes oportunidades desta articulação intrínseca entre técnica construtiva e projeto. Com esta possibilidade, surgem os edifícios regrados, definidos segundo os mesmos parâmetros, mas que estariam longe de um modelo de repetição modernista. O impacto imediato na gestão deste processo de projeto é sua alteração para permitir o surgimento de um processo colaborativo porque, tanto a arquitetura como as engenharias, possuem escopos no início do projeto. Portanto, as novas ordens morfológicas passarão a ser mais complexas porque trazem mais variáveis articuladas entre si para os estágios iniciais da antiga fase de concepção criadora. 
Segundo Schumacher (2014), esta nova heurística permite um critério de autocrítica e de um projeto em contínuo desenvolvimento, mas que depende de um processo aberto, sem completude e que não pode ser fechado ou determinado em uma única opção.

\section{Modelagem para a Interoperabilidade}

A forma gerada pelos plugins de modelagem algorítmica, quando aliada à formulação de arquiteturas, depende de uma relação intrínseca com o processo de produção do objeto. Em alguns casos ainda é necessário um processo elaborado de execução. Ainda na fase de planejamento ou projeto, a aliança a outros software de análise tem como objetivo inicial a otimização de recursos, mas também permite que este processo deixe de ser hermético, uma vez que ele pode ser aberto pela codificação da geometria até então determinada por critérios subjetivos e que passa a ser formada por interferências não geométricas. Neste caso é necessário reavaliar o processo de projeto à luz da interoperabilidade.

O conceito de interoperabilidade, entre as diferentes interpretações existentes define uma comunicação de múltiplos níveis sem interpretação ambígua dos dados a serem trocados entre sistemas. Nos últimos anos, ao invés de criar elementos padrão para resolução da interoperabilidade, foram definidas as linguagens IFC e a XML para permitir 0 trânsito de dados entre programas a partir de bases consistentes que minimizem ou até impeçam a perda de informação. Porém, é importante observar que esta estruturação dos dados determina uma interoperabilidade semântica que tem como objetivo impedir a perda de dados ou da informação. Este tema já tem sido debatido ou até solucionado em alguns casos, mas a preocupação sobre o estudo da interoperabilidade no processo de concepção deve ser alvo de uma análise mais aprofundada de modo a permitir a completa definição de um sistema interoperável evitando conflitos estruturais como a criação de um mesmo atributo ou conceito em duas estruturas de dados distintas.

De acordo com Tolk e Muguira (2003), apesar de usar uma mesma ontologia ou modelo (de dado) de referência, mesmo que seja padronizado, não é suficiente para determinar uma interoperabilidade conceitual. O mesmo objeto e com o mesmo sentido, pode ser usado de modo completamente diferente na simulação de alguns sistemas. Para evitar este problema é necessário evitar variações estruturais na geração das informações, ou seja, deve haver um alinhamento dos dados dinâmicos - dados que são construídos a partir de interferência externa. A solução recomendada é a criação de um comportamento que seja visível entre os integradores do sistema (Tolk e Muguira, 2003). Entendemos que a integração entre os sistemas pode ser interpretada dentro da modelagem gerativa a partir da interação entre softwares e o BIM, e que neste artigo serão apresentados pelo par Dynamo - Revit@. Neste caso, a criação de um comportamento visível é real porque o processo de modelagem depende de uma lógica de programação representada pelas conexões entre nós dos scripts destes programas.

A questão que permanece é sobre o nível de interoperabilidade necessário para estes processos que dependem de dados externos para serem transcodificados em elementos constituintes de uma morfologia arquitetônica.

Os níveis do modelo conceitual de interoperabilidade (LCIM) apresentados por Tolk e Muguira (2003); Tolk, Diallo e Turnitsa (2007), diferenciam cada condição para definição de um sistema conceitualmente interoperável. Neste panorama, estruturado em seis níveis de interoperação entre sistemas, o quinto nível é especialmente determinado pela possibilidade de ação dinâmica entre os sistemas. Neste estágio, é necessário que as mudanças, asserções, variações e constantes que afetem a troca de dados ocorridas neste conjunto sejam compreendidas ao longo do tempo de modo que, a partir da interação entre componentes dos sistemas, seja possível tirar vantagem desse relacionamento. Aqui podemos compreender a mudança de estado de algumas definições do projeto. De acordo com os autores, quando é necessária a análise ou produção de efeitos, este nível de interoperação determina o sucesso e eficiência da atividade.

Caso o sistema atinja este quinto nível, ainda surge outra restrição para definição de sua interoperabilidade. O problema comum que impede esta adequação conceitual final é uma falha na modelagem ou simulação de constantes. Como o modelo inicial é ator de uma realidade ou afeta um segundo modelo que será o hospedeiro do primeiro, as partes componentes da interação devem ser plenamente estabelecidas de modo a não perder a interação entre as relações de modo a garantir a interoperabilidade completa. Então, neste último nível de abstração, o modelo conceitual de trânsito das informações depende também da sua possibilidade ser compreendido e manipulado por outros agentes. Este modelo de organização das informações é muito rígido e definido pelo software Revit@, que já é estruturado segundo uma lógica BIM para produção de informações construtivas e compartilháveis com outros programas, mas a relação entre o par de software RevitDynamo abre a possibilidade de operação na própria lógica de produção de um modelo geométrico e de informações da construção.

Existem diversas vantagens na criação de modelos conceituais de interoperabilidade e que são previstas na maioria dos programas que se propõem à interação entre outros sistemas, mas a adoção de um sistema gerativo dentro desta estrutura relativamente hermética de produção de informações (presas pela lógica de programão do programa), rompe com estes modelos individuais e permite ao usuário elaborar uma lógica própria de cada projeto. Apesar desta abertura, o fato de representar em gráfico ou texto esta estrutura de parâmetros permite a externalização do processo, gerando a possibilidade de avaliar as ideias e propor mudanças inovadoras na concepção de informações, tanto geométricas como construtivas.

Em todo caso, ainda é necessário operar sobre a lógica federada de modo que uma base de dados local alimente outra que lhe é superior até ser reunida sobre um modelo federado capaz de reunir outras bases externas e até mesmo ser alimentado por elas. Nas próximas análises será 
apresentada a necessidade de uma lógica federada para o processo de modelagem a partir de critérios comuns.

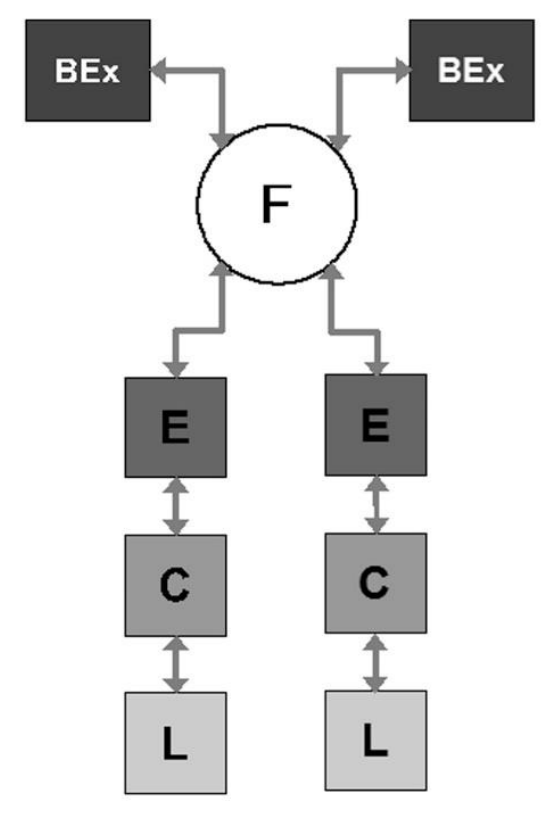

Figura 1: Esquema de base de dados federadas entre bases Locais, Componentes e de Exportação, centralizados em um modelo Federado, dentro de uma abordagem bottom-up, que alimenta e é alimentado por Bases Externas. Adaptado de Tolk, Diallo e Turnitsa, (2007).

No contexto BIM, a interação de dados construtivos com parâmetros de projeto, sejam eles formais, orçamentários ou quantificáveis, e ainda um conjunto de análises estruturais e de otimização da performance do edifício em um único conjunto de modelos de informações da construção eleva o processo de projeto para outro panorama consolidando diversas práticas atuais, mas também gerando novas atribuições no projeto.

Tendo como premissa que a articulação entre modelo algorítmico e BIM é um meio para consolidação da potência tectônica deste processo, os estudos desenvolveram mecanismos de importação, exportação e interação de parâmetros geométricos e construtivos a partir da inserção da informação em distintos suportes do programa como famílias, projetos e componentes. As análises a seguir indicam métodos de trabalho diferentes nos quais foi possível perceber a dependência de grau de informações entre modelos.

\section{Métodos e Análises}

\section{Caso 1 -Torre Eólica Treliçada}

A pesquisa desenvolvida por Borges e Fakury (2015) representa 0 processo de modelagem integrada à condicionantes físicas de dimensionamento da estrutura tubular de suporte de uma torre eólica de 140 metros de altura.
As condicionantes físicas partiram de cargas e tensões orientadas pelo nível máximo de stress da estrutura permitindo a otimização a partir algoritmos desenvolvidos pelos autores. No processo apresentado, a forma é gerada em um primeiro momento a partir de critérios estéticos nos software Rhinoceros e Grashopper, mas com sua geometria ajustada segundo um nível de stress máximo. A partir deste resultado foram desenvolvidas outras análises que determinaram a quantidade de tubos de acordo com o catálogo da indústria. A segunda otimização ocorreu por meio de um código programado em Visual Basic dentro do Grasshopper e que apresentou o menor número de peças necessárias para a construção da estrutura, mas que opera

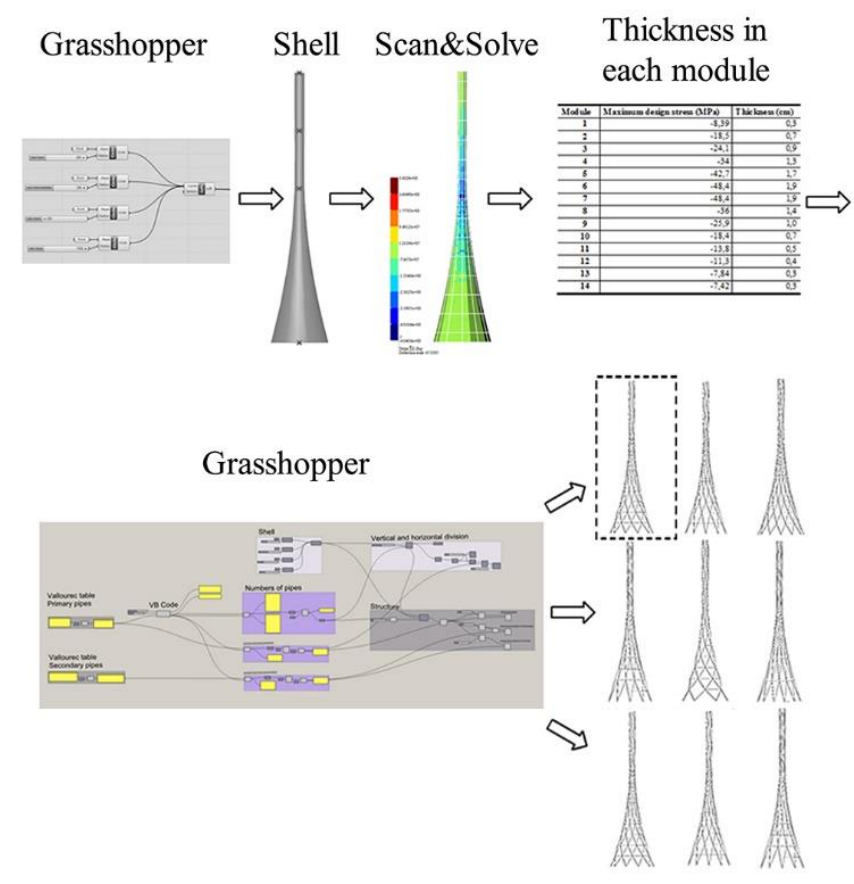

distante de um modelo BIM.

Figura 2: Fluxo de trabalho desenvolvido pela pesquisa (Borges e Fakury, 2015). Cortesia dos autores.

Como consequência desta pesquisa os autores apontam que o processo de gênese é integrado e a fronteira entre áreas de atuação dos profissionais é reduzida pela característica do processo gerativo, dependente de parâmetros construtivos em fases iniciais de concepção. Apesar desta vantagem, o processo de projeto experimentado poder gerar informações sobre peças, seu escopo não determinou um modelo BIM.

\section{Caso 2 - Estrutura paramétrica}

Outro projeto analisado que objetiva a otimização de uma estrutura foi encontrado em Lee et al (2015). No caso de construções de maior complexidade, como apresentado pelo autor, é necessário um modelo de gestão do projeto que permita a centralização da informação, tanto para coordenação do processo como informação constante do processo, desde o projeto à manutenção do edifício. $\mathrm{Na}$ modelagem BIM, é oferecido o acesso a maquetes, análises de interferência, simulações e estimativas de quantidade de 
materiais diretamente vinculadas ao custo das soluções. No projeto mencionado por Lee et al (2015) a solução encontrada para produzir a forma final da estrutura foi dividir a fase de concepção em dois grupos de acordo com as partes que compõem a geometria - estrutura e ancoragem. Como conclusão, a pesquisa aponta a possibilidade da modelagem algorítmica em um processo BIM, uma vez que o modelo paramétrico permite o reuso dos dados iniciais, i.e. parâmetros condicionantes do projeto, para diversos processos como fabricação, manutenção e construção.

\section{Caso 3 - Integração Revit®-Dynamo}

Neste primeiro exemplo foi desenvolvida uma fachada segundo um script determinado pelo plugin Dynamo Studio 0.8.2 de modo a ser incorporada em uma superfície de um projeto iniciado no software Revi $\AA^{\circledR} 2015$. A proposta é similar à alternativa amplamente difundida pela solução dada pelo escritório Aedas Architects no projeto das Torres Al Bahar em 2012, e faz parte da criação de um módulo responsivo e independente que é modificado de acordo com a posição do sol em relação à fachada estudada.

Esta resposta é determinada por uma análise entre dois vetores, a normal do plano base do módulo e a direção da luz solar, transcodificada em um vetor que é alterado pelas condições de insolação disponíveis no Revit®.

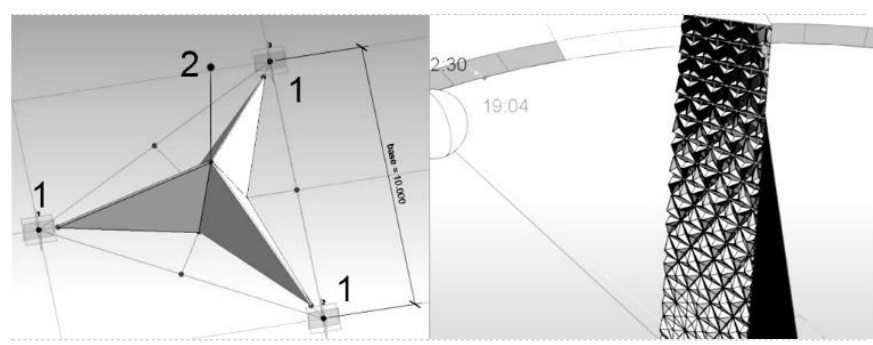

Figura 3: À esquerda, arquivo modelado em RFA (Revit Family). 1vínculos do módulo à superfície. 2- normal do plano de referência. À direita, projeto do edifício em .RVT(Arquivo Revit Project). A fachada recebe o módulo vinculado à subdivisões da superfície.

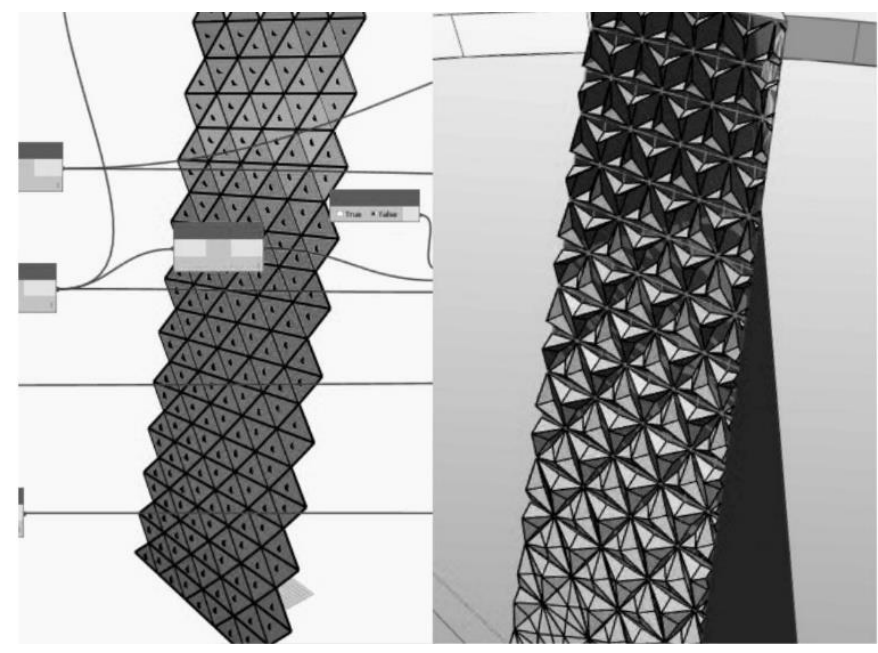

Figura 4: Superfície da fachada analisada a partir do ângulo de incidência do sol. A informação externa ao projeto e que alimenta a análise é dada pelas coordenadas existentes no Revit, enquanto a análise em gradiente de cores foi construída no Dynamo Studio.

O sol é, então, transcodificado em um painel da fachada que reage de acordo com a variação do parâmetro altura através de um mapa de insolação otimizando a penetração da luz. Neste caso, é criada a articulação da fachada com a eficiência do material-abertura em uma representação dessa sua diferenciação de acordo com a direção do sol e a carga térmica que é projetada sobre a superfície.

\section{Conclusão}

O primeiro caso, a Torre eólica treliçada determina uma geometria otimizada estruturalmente, mas pela estrutura dos softwares utilizados e pelo escopo do projeto, não determinou um modelo BIM. Dentre os softwares de modelagem gerativa (ou generativa) em CAAD, o Grasshopper é mais difundido, mas até a conclusão desta pesquisa apresentava falta de interação de seus modelos com a plataforma BIM. Atualmente já existe a iniciativa da parceria entre as empresas McNeel com a Graphisoft no sentido de criação de um plugin que auxilie a interoperabilidade entre sistemas. Dentre os outros softwares gerativos restantes, o Dynamo Studio inicia a interação BIM com o Autodesk Revit, mas por ainda ser recente, possui poucos plugins de suporte, ao contrário do Grasshopper cuja maior vantagem reside na sua linguagem aberta e amplo repertório de plugins para análise das geometrias.

Na experiência realizada por esta pesquisa, em comparação com os casos analisados, é possível apontar possíveis problemas na adoção de uma prática de modelagem livre, alheia à estrutura das operações inerentes à prática BIM apesar de não participarem de um projeto de grande complexidade e em um ciclo ativo de produção completa em BIM (intercâmbio de arquivos e projeto simultâneo, colaborativo).

Nesta primeira análise é possível prever que a importação de famílias de um projeto dentro do Dynamo para sua alteração por inserção de condições e critérios de análise internos aos scripts adotados deve ser orientada segundo a matriz federada uma vez que, em projetos complexos, a sua importação para o Dynamo pode gerar listas extremamente longas e desnecessárias devido à quantidade de componentes utilizados naquele projeto. Essa ação ainda tem maior impacto quando o modelo está associado a um modelo centralizado, compartilhado por equipes. Neste caso, esta pesquisa permite afirmar uma prática mais eficiente ao indicar a necessidade de federação da base de dados e, consequentemente, os componentes da construção. No caso apresentado, a fachada deveria ser tratada como um arquivo à parte que seria inserido como referência ou como uma família dentro de um projeto maior. Neste caso, toda e qualquer modificação ou otimização de seus parâmetros no Dynamo, geraria pouco ou nenhum impacto na eficiência do projeto compartilhado porque a fachada estaria estruturada como um elemento independente.

A partir dos níveis de interoperabilidade apresentados por Tolk, Diallo e Turnitsa, (2007), foi percebido que a versão 
utilizada, Dynamo 0.9.2, não suporta uma interoperabilidade dinâmica porque a operação sobre parâmetros ocorre de modo unidirecional, ao contrário do que era esperado. Dentro da modelagem gerativa do Dynamo, os dados de entrada podem ser importados do Revit, alterados pelo Dynamo e realimentados novamente no Revit. A modificação do elemento enquanto composição paramétrica do Dynamo pode ser visualizada no Revit como um elemento temporário, ainda virtual, mas depois de finalizado o processo, aquele elemento se torna uma instância do projeto e qualquer modificação ou complementação da informação que ocorra na plataforma do Revit só pode transferir seus dados para o Dynamo ser for novamente importada por meio de outro nó. Desta forma as modificações não acontecem em tempo real no par RevitDynamo, somente no seu sentido inverso. Isso coloca o plugin Dynamo Studio na posição de constructo de informações de entrada para o projeto e não pode ser entendido como um mecanismo algorítmico em tempo real, dinâmico.

Apesar desta crítica ainda reside uma potência transformadora porque ele permite a produção de um processo tectônico, uma vez que o plugin expõe para modificação uma série de parâmetros construtivos das geometrias que lhe são transferidas.

Os plugins de modelagem gerativa são mecanismos de programação, ou seja, determinam dados de entrada que serão processados por outro mecanismo com intuito de gerar um dado de saída que pode ou não realimentar o processo ou outro a ele vinculado. A inovação do processo reside na sua didática, ou seja, a interface de programação visual que apresenta os recursos pelos quais é possível pensar uma nova gênese da forma e sua relação com outros elementos.

Com a percepção do potencial de interoperabilidade entre programas e seu incentivo pelas empresas proprietárias destes grupos de programas, esta parametrização atinge outro patamar uma vez que os dados criados em um programa interferem não apenas os critérios geométricos ou de automação de rotinas, mas alimentam e condicionam algoritmos em outros softwares de análise como metadados. Neste cenário de interação entre softwares e dados, é necessário ficar atento para critérios de análise do processo e de balizamento em direção a boas práticas de projeto. Como esta prática é complexa, dependemos de padrões de modelagem para desenvolver modelos mais consistentes.

\section{Agradecimentos}

Agradecemos ao Conselho Nacional de Desenvolvimento Científico e Tecnológico (CNPq), por ter financiado a pesquisa da qual este artigo faz parte.

\section{Referências}

BENTSCHEFF, I.; GENGNAGEL, C. (2010). Towards Teaching Generative Design in Architecture. In: Ceccato C. et al. Advances in Architectural Geometry. Springer Wien New York, Viena.(pp 113-128)

BORGES, M; FAKURY, R.H. (2015). Structural design based on performance applied to development of a lattice wind tower. In CELANI, G. et al. (eds.): CAAD Futures 2015, CCIS 527, pp 255-271.
LEE, S. et al. (2015). 3D digital fabrication and erection technologies for prefabricates bridges. In MAHDJOUBI, L., BREBBIA, C.A. ,LAING, R.(eds). Building Information Modeling (BIM) in Design, Construction and Operations, (pp.3-8). Boston: WITPress

SCHUMACHER, P.. (2008). Smart Work - Patrik Schumacher on the Growing Importance of Parametrics, RIBA Journal, 2008, September. Disponível

em

http://www.patrikschumacher.com/Texts/Design\%20Research\%20at \%20Zaha\%20Hadid\%20Architects.htm

SCHUMACHER, P.. (2012) Tectonics - The Differentiation and Collaboration of Architecture and Engineering. Contribution to the catalogue/book 'Stefan Polonyi - Bearing Lines - Bearing Surfaces', published by MAI - Museum für Architektur und Ingenieurkunst NRW e.V., Ed. Ursula Kleefisch-Jobst et al., Edition Axel Menges, Stuttgart/London. Retirado de

http://www.patrikschumacher.com/Texts/Tectonics\%20-

\%20The\%20Differentiation\%20and\%20Collaboration\%20of\%20Archi tecture\%20and\%20Engineering.htm

SCHUMACHER, P.. (2016) Design Parameters to Parametric Design. In: The Routledge Companion for Architecture Design and Practice: Established and Emerging Trends. Edited by Mitra Kanaani and Dak Kopec, Routledge,Taylor and Francis, New York. Retirado de http://www.patrikschumacher.com/Texts/Design\%20Parameters\%20t 0\%20Parametric\%20Design.html

STAPLETON, K. A. J.; GLEDSON, B. J.; ALWAN, Z.. (2014) Understanding technological interoperability through observations of data leakage in Building Information Modelling (BIM) based transactions. In: Proceedings of the 32nd eCAADe Conference Volume 2, Department of Architecture and Built Environment, Faculty of Engineering and Environment, Newcastle upon Tyne, England, UK, 10-12, pp. $515-524$

TOLK, A., J.A. MUGUIRA, J.A., (2003) "The Levels of Conceptual Interoperability Model (LCIM)," Proceedings IEEE Fall Simulation Interoperability Workshop, IEEE CS Press.

TOLK, A., DIALLO, S., TURNITSA, C.. (2007) Applying the Levels of Conceptual Interoperability Model in Support of Integratability, Interoperability, and Composability for System-of-Systems Engineering. Journal of Systemics, Cybernetics and Informatics. v 5 n5, 65-74. 